\title{
A Xanthomonas oryzae type III effector XopL causes cell death through mediating ferredoxin degradation in Nicotiana benthamiana
}

Wenxiu Ma ${ }^{1,2}$, Xiameng Xu1 ${ }^{1}$ Lulu Cai ${ }^{1,3}$, Yanyan Cao ${ }^{1}$, Fazal Haq ${ }^{1}$, James R. Alfano ${ }^{1,4}$, Bo Zhu' ${ }^{1}$, Lifang Zou ${ }^{1}$ and Gongyou Chen ${ }^{1 *}$ (i)

\begin{abstract}
Plant pathogenic bacteria engage in an ongoing struggle for survival. Gram negative phytopathogenic prokaryotes deploy type III secretion system to deliver effector proteins into plant cells to manipulate the host cellular environment. In many cases, the targets of these effector proteins are unknown. The bacterial blight pathogen, Xanthomonas oryzae pv. oryzae (Xoo), causes a devastating disease in rice and the Xoo-rice interaction is an example of the ongoing evolutionary battle between plants and pathogens. In this study, we show that an effector XopL from Xoo exhibits E3 ubiquitin ligase activity and induces cell death in the nonhost Nicotiana benthamiana (Nb). Through yeast two-hybrid, co-immunoprecipitation, and biomolecular fluorescence complementation assays, it was shown that XopL interacts with ferredoxin (NbFd), an electron transport molecule in plant chloroplasts. XopL induced defense-related response in $\mathrm{Nb}$ and specifically targeted NbFd for ubiquitination and ubiquitin-mediated degradation, a process that increased the production of reactive oxygen species (ROS). This study demonstrates the ubiquitination and degradation of $\mathrm{NbFd}$ as a result of XopL activity, which further illustrates the tremendous functional diversity in Xanthomonas effector proteins.
\end{abstract}

Keywords: Xanthomonas oryzae pv. oryzae, XopL, Cell death, Ferredoxin, Degradation, ROS, Ubiquitination

\section{Background}

Plants are continually bombarded by abiotic and biotic stressors, including phytopathogenic viruses, fungi, nematodes, and bacteria. The bacterial genus Xanthomonas includes over 30 pathogenic species that cause diseases on many economically-important crops including banana, Brassica spp., cassava, citrus, rice, tomatoes, and tobacco (Schornack et al. 2013; Jacques et al. 2016). Protein secretion systems (e.g. Types I-VI, Sec, TAT) play important roles in the successful colonization of host plants by phytopathogenic bacteria, including

\footnotetext{
* Correspondence: gyouchen@sjtu.edu.cn

${ }^{1}$ School of Agriculture and Biology/State Key Laboratory of Microbial Metabolism, Shanghai Jiao Tong University, Shanghai 200240, China Full list of author information is available at the end of the article
}

Xatnhomonas spp. (Kay and Bonas 2009; Büttner 2016). Many pathogenic xanthomonads rely on the type III secretion system (T3SS) to successfully invade and multiply inside plant tissues (Kay and Bonas 2009). The T3SS translocates type III effector (T3E) proteins into host cells, which can facilitate pathogen-mediated manipulation of plant cellular pathways and subsequent disease or trigger plant defense responses to thwart the infection process (Dean 2011; Lee et al. 2013).

During co-evolution with pathogens, plants developed a multi-layered immune system to provide protection from pathogen ingress (Dodds and Rathjen 2010). The first layer of defense involves the recognition of pathogen-associated molecular patterns (PAMPs), such as bacterial flagellin, peptidoglycan, and chitin. The

(c) The Author(s). 2020 Open Access This article is licensed under a Creative Commons Attribution 4.0 International License, which permits use, sharing, adaptation, distribution and reproduction in any medium or format, as long as you give appropriate credit to the original author(s) and the source, provide a link to the Creative Commons licence, and indicate if changes were made. The images or other third party material in this article are included in the article's Creative Commons licence, unless indicated otherwise in a credit line to the material. If material is not included in the article's Creative Commons licence and your intended use is not permitted by statutory regulation or exceeds the permitted use, you will need to obtain permission directly from the copyright holder. To view a copy of this licence, visit http://creativecommons.org/licenses/by/4.0/ 
recognition of PAMPs is modulated by plant-encoded pattern-recognition receptors (PRRs), which include receptor-like kinases and proteins that are often located in plant cell membranes. The recognition of PAMPs results in defense known as PAMP-triggered immunity (PTI) and the activation of responses that interfere with pathogen invasion and colonization (Schwessinger and Ronald 2012; Wu and Zhou 2013). In addition to PTI, plants have evolved another layer of defense known as effector-triggered immunity (ETI); this is activated via the direct or indirect recognition of pathogen effectors by plant resistance $(R)$ genes and is associated with the hypersensitive response (HR), a form of programmed cell death that limits pathogen multiplication (Wu et al. 2014). Thus, T3Es may either trigger the plant defense response (Khan et al. 2016) or manipulate plant cellular pathways in ways that benefit the bacterial pathogen (Büttner 2016).

Plants also evade many potential pathogens due to nonhost resistance (NHR), which is the most common form of plant resistance and is effective against a wide array of pathogens (Mysore and Ryu 2004; Fan and Doerner 2012). The mechanism of NHR is complex, which includes preformed physical barriers, secondary metabolites, and induced defense. Plant NHR reactions vary considerably, ranging from symptomless reactions to the HR and programmed cell death (Mysore and Ryu 2004; Uma et al. 2011; Senthil-Kumar and Mysore 2013). In some cases, bacterial effectors are recognized by nonhost plants, thus triggering an HR or cell death. For example, XopQ (HopQ) from Xanthomonas triggers ETI on the nonhost $\mathrm{Nb}$ (Wei et al. 2007; Schwartz et al. 2015). Recently, a resistance protein from $N b$ designated Roq1 (Recognition of XopQ1) was shown to interact with XopQ, which led to an HR (Schultink et al. 2017). Moreover, the transgenic expression of Roq1 in beet plants was sufficient to trigger recognition of XopQ, thus indicating that $R$ genes from heterogeneous plant species may improve host resistance in unrelated species (Schultink et al. 2017).

The closely-related xanthomonads, $X$. oryzae $\mathrm{pv}$. oryzae $(X o o)$ and $X$. oryzae pv. oryzicola $(X o c)$, cause bacterial blight (BB) and bacterial leaf streak (BLS) on rice, respectively (Nino-Liu et al. 2006; Zhang and Wang 2013). Like many other bacterial pathogens, successful infection by $X$. oryzae depends on the T3SS, which is necessary for its pathogenicity on host rice and the HR on nonhost $N b$. Two kinds of proteins traverse the T3SS in $X$. oryzae; these include the T3E proteins that are directly delivered into plant cells (Kay and Bonas 2009) and extracellular accessory proteins such as the harpin Hpa1 (Zou et al. 2006).

Xanthomonas T3Es are broadly categorized into two different families: TALE (transcription activator-like effectors) and non-TALE which are also called Xanthomonas outer proteins (Xop) effectors. Many Xop effectors interfere with PTI and ETI-associated plant immunity (Büttner 2016), however, only relatively few of them are well-defined in the precise cellular targets and modes of action. For example, XopP targets OsPUB44 U-box and inhibits rice immunity (Ishikawa et al. 2014). XopY (Xoo1488) inhibits rice immunity by suppressing OsCERK1-mediated phosphorylation of OsRLCK185 (Yamaguchi et al. 2013).

Recently, ubiquitination was shown to be utilized by pathogen effectors to gain a competitive edge (Ustun and Bornke 2014; Pruneda et al. 2016; Lin and Machner 2017). Ubiquitination alters the fate of a target protein, often tagging it for subsequent degradation by the $26 \mathrm{~S}$ proteasome (Ustun and Bornke 2014). A number of Xanthomonas T3Es interact or modulate the host ubiquitin-proteasome system including XopJ, XopD, XopK, XopAE, and XopL (Singer et al. 2013; Ustun and Bornke 2014; Pruneda et al. 2016; Popov et al. 2018; Qin et al. 2018). Singer et al. demonstrated that XopL from $X$. campestris pv. vesicatoria $\left(\mathrm{XopL}_{X c v}\right)$ contains E3 ubiquitin ligase activity and interacts with plant E2 ubiquitin conjugating enzymes (Singer et al. 2013). Recently, the transient expression of $\mathrm{XopL}_{X c v}$ in $\mathrm{Nb}$ caused major ultrastructural changes including the relocation of plastids to the nucleus (Erickson et al. 2018). Despite these advances in the understanding of XopL function, the precise targets of XopL have remained unclear.

In this study, we explore the role of $\mathrm{XopL}_{X o o}$ in mediating nonhost resistance in $\mathrm{Nb}$, a model plant that has been widely-used to study the function of numerous Xanthomonas T3Es including XopH, XopJ, XopQ, XopX and XopL (Thieme et al. 2007; Singer et al. 2013; Stork et al. 2015; Adlung et al. 2016; Blüher et al. 2017; Erickson et al. 2018). A yeast two-hybrid assay was used to demonstrate the interaction between XopL and ferredoxin (NbFd). The interaction of XopL and NbFd was further confirmed by co-immunoprecipitation and biomolecular fluorescence complementation assays. XopL was shown to catalyze the ubiquitination of $\mathrm{NbFd}$, which led to subsequent degradation of $\mathrm{NbFd}$ in planta and increased levels of reactive oxygen species and defense genes.

\section{Results}

\section{XopL induces cell death in Nicotiana benthamiana}

$X$. oryzae triggers strong cell death in non-host $N$. benthamiana $(N b)$. In order to find new cell death inducer(s) from $X$. oryzae, an initial screen for effectors that induce cell death in $\mathrm{Nb}$ was conducted in $\mathrm{X}$. oryzae pv. oryzicola (Xoc) RS105. Twenty-two predicted T3Es (http://www.xanthomonas.org/t3e.html) from Xoc were amplified using primers in the Additional file 1: Table 
$\mathrm{S} 1$ and transiently expressed via Agrobacterium in $\mathrm{Nb}$ leaves. In addition to the positive harpin Hpal (Zou et al. 2006), five effectors, namely, $\mathrm{XopL}_{X o c}, \mathrm{XopP}_{X o c}$, XopP2 $2_{\text {Xoc }}$, XopAA $_{X o c}$ and XopY Xoc, triggered cell death when transiently expressed in $\mathrm{Nb}$ (Fig. 1).

According to the Xanthomonas resource website (www.xanthomonas.org/t3e.html) and previous work (Singer et al. 2013), XopL is conserved in nine out of thirteen Xanthomonas strains from eight species. Thus, we cloned xopL from Xoo model strain $\mathrm{PXO}^{\mathrm{A}}{ }^{\mathrm{A}}$ and found that $\mathrm{XopL}_{X o o}$, like $\mathrm{XopL}_{\mathrm{Xoc}}$ and $\mathrm{XopL}_{\mathrm{Xcv}}$ (Singer et al. 2013), also induced cell death in $N b$, which indicates this function is conserved in Xanthomonas species. Given the fact that the bacterial blight pathogen Xoo $\mathrm{PXO}^{\mathrm{A}}$ is a model strain well-studied, we performed a study on XopL $\mathrm{X}_{\text {Xoo }}$ (XopL hereafter). XopL induced cell death at 7 days post-inoculation (dpi) when transiently expressed in $\mathrm{Nb}$ (Fig. 2a). Evans blue staining and ion leakage measurements confirmed that XopL triggered cell death in $\mathrm{Nb}$ (Fig. $2 \mathrm{~b}$ and Additional file 2: Figure S1). Localization of XopL:YFP fusions indicated that XopL was primarily localized to the cytoplasm (Additional file 3: Figure S2).

To determine whether XopL inducing-cell death was accompanied with defense responses in $N b$, we examined the expression of several defense-related genes in $\mathrm{Nb}$ agroinfiltrated with $\mathrm{pHBxopL}$ and $\mathrm{pHB}$ (empty vector control). Highly expressed genes include the pathogenesis-related $(P R)$ genes PR4, PR5 and PR10, the HR marker gene HIN1 (Gopalan et al. 1996), the master immune regulatory gene NPR1, PAMP-triggering immunity-related gene PTI5 (Nguyen et al. 2010) and elicitor-inducible cytochrome P450 CYP71D20 (CYP) (Lacombe et al. 2010) (Fig. 2c). Furthermore, the expression of xopL resulted in $\operatorname{ROS}\left(\mathrm{H}_{2} \mathrm{O}_{2}\right)$ accumulation; this was obvious at $3 \mathrm{dpi}$ and was more intense at $6 \mathrm{dpi}$ (Fig. $2 \mathrm{~d}$, e). Thus, XopL induces cell death when transiently expressed in $N b$, and this is accompanied by the defense genes activation and ROS accumulation.

\section{XopL interacts with $\mathrm{Nb}$ ferredoxin protein}

To better understand the mechanistic basis for XopLmediated defense in $N b$, XopL-interacting proteins were identified from an $\mathrm{Nb}$ cDNA library (Sun et al. 2015) of mature leaves using full-length XopL as bait (pGBKT7xopL, Additional file 4: Table S2) in a yeast two-hybrid assay. A total of 56 positive colonies were initially identified, which represented 11 different genes. To further confirm positive interactions, we swapped the bait and prey roles. The $x o p L$ gene was cloned into pGADT7 and used as prey; the coding regions of the 11 different genes were cloned into pGBKT7 as baits and co-transformed into S. cerevisiae AH109 with pGADT7-xopL. Clones encoding ferredoxin ( $\mathrm{NbFd}$, AKQ98361) but not empty BD interacted with XopL (Fig. 3b and Additional file 5: Figure S3). According to co-immunoprecipitation (CoIP) results, we found XopL co-immunoprecipitated with NbFd-YFP but not YFP (Fig. 3c), which suggests an interaction between NbFd and XopL in planta.

We conducted BiFC assays to further explore the interaction between XopL and NbFd. Confocal microscopy indicated that the co-expression of XopL:YN and $\mathrm{NbFd}$ :YC generated yellow fluorescent signals in the cytoplasm (Fig. 3d, top row, YFP panel), which were absent in the controls. These results confirmed that XopL interacts with $\mathrm{NbFd}$ in the cytoplasm.

Ferredoxin is encoded by nuclear DNA and transported into chloroplast via the chloroplast addressing peptide at the N-terminus end (Pilon et al. 1992). Localization assays of the NbFd:YFP fusion indicated

\begin{tabular}{|c|c|c|c|}
\hline Effector & Cell death & Effector & Cell death \\
\hline $\mathrm{XopC}_{\text {Xoc }}$ & & XopW $_{\text {xoc }}$ & \\
\hline$X_{\text {XopF }}$ & & $X_{\text {XopX }}$ & \\
\hline Xopl $_{X o c}$ & & XopY $_{\text {Xoc }}$ & Yes/++ \\
\hline $\mathrm{XopK}_{\text {xoc }}$ & & $X_{\text {Xpp }} Z_{\text {Xoc }}$ & \\
\hline XopL $_{\text {Xoc }}$ & Yes/++ & XopAA $_{\text {Xoc }}$ & Yes/+ \\
\hline XopN $_{\text {Xoc }}$ & & XоpAB $_{\text {Xoc }}$ & \\
\hline $\mathrm{XopO}_{\text {Xoc }}$ & & XopAE $_{\text {xoc }}$ & \\
\hline XopP1 $1_{\text {Xoc }}$ & Yes/+++ & XopAF $_{X o c}$ & \\
\hline XopP $2_{\text {xoc }}$ & Yes/+++ & ХорАК $_{\text {хос }}$ & \\
\hline$X o p Q_{X o c}$ & & $\mathrm{HpaA}_{x o c}$ & \\
\hline XopR $_{\text {Xoc }}$ & & $\mathrm{Hpa1}_{\text {Xoc }}$ & Control/+++ \\
\hline$X_{o p V_{X o c}}$ & & & \\
\hline
\end{tabular}

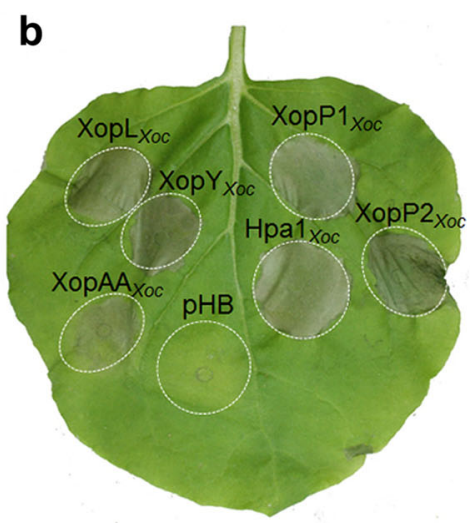

Fig. 1 Cell death responses induced by T3Es of X. oryzae pv. oryzicola RS105. a The predicted T3Es and their abilities to trigger cell death in Nb. T3Es were transiently expressed in tobacco leaves mediated by Agrobacterium. Harpin protein Hpal was used as positive cell death inducer. +++ , ++ , and + indicate cell death appeared at 4, 7, and 10 dpi, respectively. b Cell death phenotypes induced by effectors. Picture was taken at $10 \mathrm{dpi}$ 

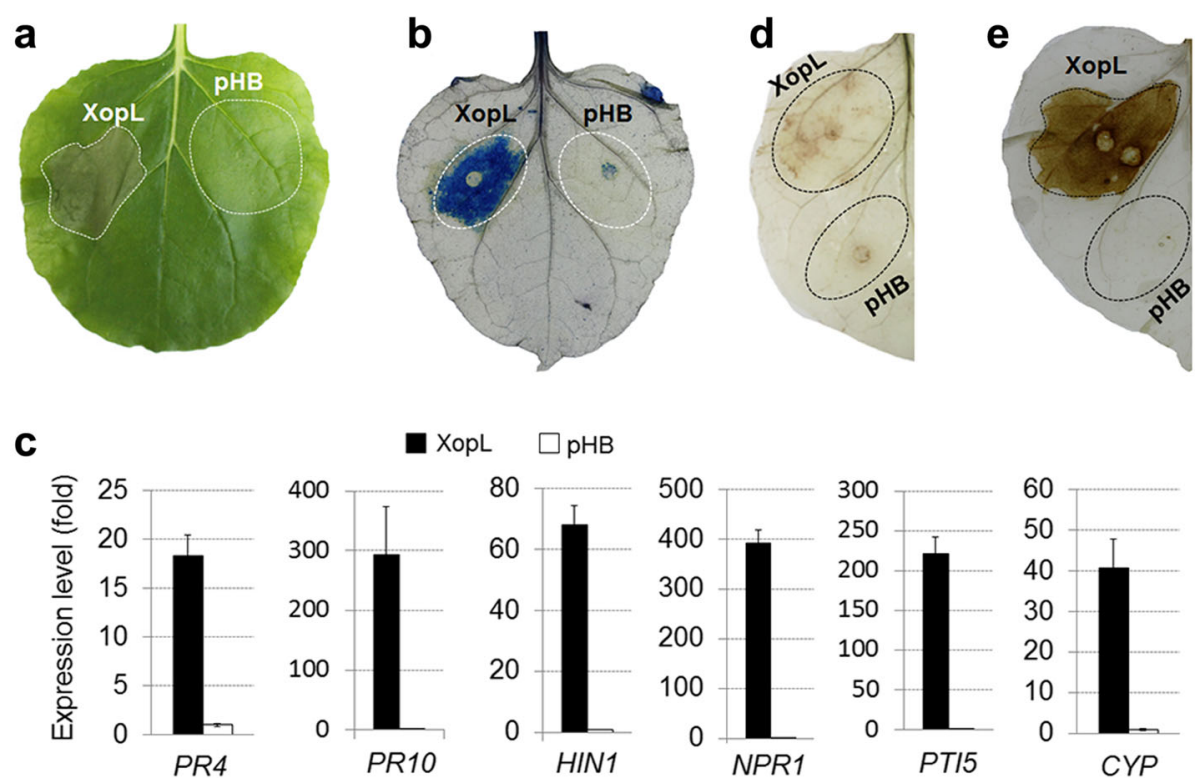

XopL $\square \mathrm{pHB}$
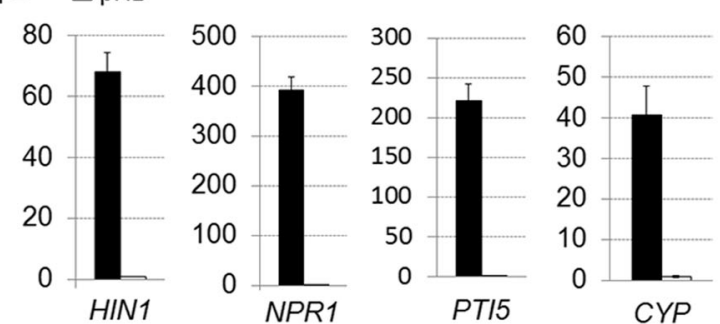

Fig. 2 Analysis of cell death and defense responses in Nb induced by XopL from Xoo. a Phenotype of infiltrated leaves at 7 dpi. Experiments were repeated five times with similar results. $\mathbf{b}$ Detection of cell death using Evans blue. The inoculated leaves were stained and photographed at $7 \mathrm{dpi}$. Picture was representative of three repeats. c Expression of defense-related genes at $4 \mathrm{dpi}$ in $\mathrm{Nb}$ plants transformed with pHB-xopL or empty pHB. Mean values $\pm \mathrm{SD}(n=3)$ are shown. Results are representative of three independent replicates. $\mathbf{d}$, e Detection of ROS $\left(\mathrm{H}_{2} \mathrm{O}_{2}\right)$ accumulation at 3 and $6 \mathrm{dpi}$, respectively, using diaminobenzidine (DAB) staining. Experiments were performed three times with the same results

that most of the fluorescence appeared in chloroplast (Additional file 3: Figure S2, blue arrows); however, faint fluorescent signals were also observed in the cytoplasm (Additional file 3: Figure S2, red arrows), presumably during the transport of NbFd to the chloroplast. NbFd contains the $\mathrm{N}$-terminal signal peptide for import into the chloroplast (residues 1-51) (Holbrook et al. 2016), whereas the C-terminal functional domain contains the conserved [2Fe-2S] iron-sulfur active center and the four conserved cysteine residues that catalyze electron transfer (Hanke and Mulo 2013) (Fig. 3a). To clarify which region of NbFd interacts with XopL, we deleted the chloroplast-addressing peptide of $\mathrm{NbFd}$, generating $N b F d \Delta 2-51$. $N b F d \Delta 2-51$ interacted with XopL (Fig. $3 \mathrm{~b})$, indicating that the XopL-interacting region of NbFd is likely located in the C-terminal portion. These results were supported by BiFC analysis, in which XopL:YN and $\mathrm{NbFd} \Delta 2-51: \mathrm{YC}$ were co-expressed; the interaction of XopL:YN and NbFd $\Delta 2-51-Y C$ resulted in a strong fluorescent signal (Fig. 3d, second row, YFP panel).

XopL $_{X o o}$ shares $74.24 \%$ amino acid identity with $\mathrm{XopL}_{X c v}$ (Additional file 6: Figure S4) that contains Nterminal leucine-rich repeats (LRR) and a C-terminal E3 ubiquitin ligase domain designated as the XL-box (Singer et al. 2013); these regions are also highly conserved in $\mathrm{XopL}_{X o o}$ (Fig. 3a; Additional file 6: Figure S4bd). To determine which region(s) of XopL interacts with $\mathrm{NbFd}$, we designed constructs containing either the LRR or XL domain of XopL. Both the two domains of XopL interacted with $\mathrm{NbFd}$, however, yeast strains coexpressing XopL-LRR/NbFd or XopL-XL/NbFd failed to grow on SD/-Leu-Trp-His-Ade medium when diluted 1000-fold (Fig. 3b). Our results suggest that NbFd interacts with both the LRR and XL domains of XopL; however, the affinity of $\mathrm{NbFd}$ to these two domains is lower than that to the full-length of XopL (Fig. 3b).

\section{XopL mediates the ubiquitination and degradation of $\mathrm{NbFd}$}

The affinities of XopL, and the E3 ligase domain in XopL for NbFd led us to investigate whether XopL ubiquitinates $\mathrm{NbFd}$. To test this hypothesis, an in vitro ubiquitination assay was conducted using the Ubiquitin Conjugation Kit K-960 (Boston Biochem, Cambridge, MA, USA). Anti-GST antibody was used to indicate the presence of GST:XopL protein. Results showed that single band was detected in each reaction system containing GST:XopL (Fig. 4a, bottom channel; Additional file 7: Figure S5), which means that no modification occurred in GST:XopL. Then anti-ubiquitin antisera was used to detect the polyubiquitination reaction. Polyubiquitin smear $\geq 90 \mathrm{kDa}$ was present in lanes 1 and 2, while was absent from lanes lacking the E1 + E2 enzymes (Fig. 4a, middle panel), indicating that, in combination with E1 and E2, XopL could catalyze the formation of polyubiquitin molecules. When probed with anti-His antibody to detect NbFd:His protein, multiple large bands $(\geq 90$ $\mathrm{kDa}$, see immuno-reactive smear) were detected only in 


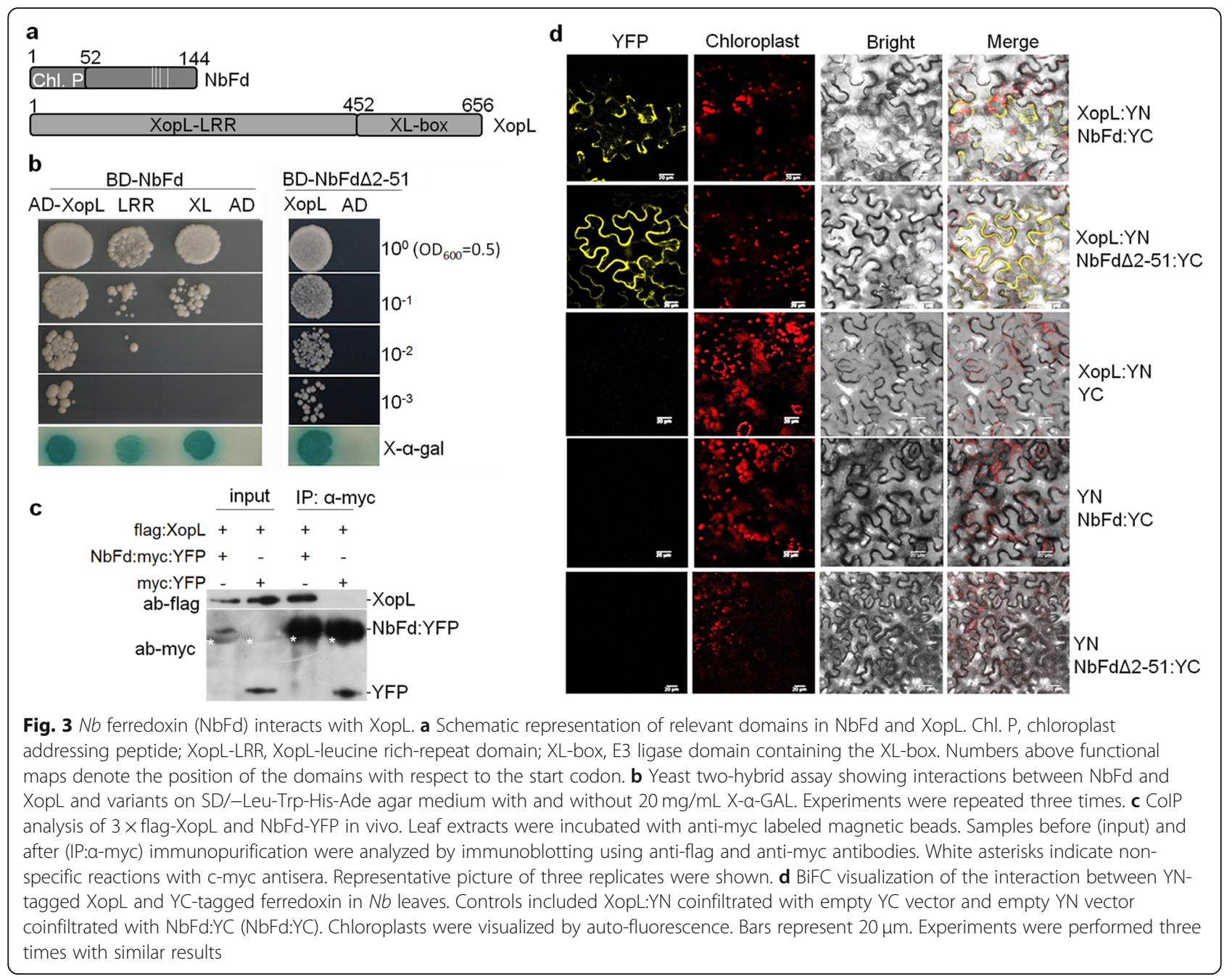

the lane containing all reaction components (Fig. 4a, top panel, lane 1), suggesting the modification and possible formation of polyubiquitinated NbFd.

Next, we investigated whether XopL mediates the degradation of $\mathrm{NbFd}$ in planta by transiently co-expressing NbFd:YFP and 3xflag:XopL or empty vector (pHB). By comparing the blotted NbFd:YFP intensity, we found that co-expression with XopL led to reduced accumulation of NbFd:YFP at 2, 3 and 4 dpi, with the lowest accumulation amount at $4 \mathrm{dpi}$ (Fig. $4 \mathrm{~b}$, top panel). The NbFd levels were similar when NbFd:YFP was incubated with pHB (Fig. 4b, top panel, lanes 1, 3 and 5). The levels of actin, the internal control, remained similar regardless of the presence or absence of XopL (Fig. 4b, bottom panel). These results suggested that XopL modulates degradation of $\mathrm{NbFd}$. However, the levels of $\mathrm{NbFd}$ remained unaltered in the presence of XopL-LRR, regardless of time point (Fig. 4c, top panel). To exclude the possibility that differences in mRNA levels resulted in altered protein levels, we checked the transcription of $N b F d-Y F P$ in infiltrated regions of $\mathrm{Nb}$ leaves by RT-PCR tests. Both
NbFd-YFP and EF1 $\alpha$ (endogenous control) expression levels were similar at each time point (Fig. $4 \mathrm{~b}, \mathrm{NbFd}$ $Y F P$ and $E F 1 \alpha$ panels), indicating that the difference in NbFd:YFP accumulation was not due to differential expression. To confirm that NbFd was degraded by the ubiquitin-proteasome system, we utilized MG132, a proteasome inhibitor that can reduce the degradation of ubiquitin-conjugated proteins. When MG132 was infiltrated into $\mathrm{Nb}$ leaves expressing NbFd-YFP and XopL, $\mathrm{NbFd}$ degradation was inhibited (Fig. 4d, ab-myc panel). Collectively, our results imply that XopL mediates the ubiquitination and subsequent degradation of NbFd.

In addition, we observed large immuno-reactive XopL and XopL-LRR bands when probed with anti-flag antibody at 2, 3 and 4 dpi in $N b$ (Fig. 4b, c, second panel, lanes 2, 4 and 6), which suggested that a modified form(s) of XopL was also present.

\section{Silencing of $\mathrm{NbFd}$ results in ROS accumulation}

To further investigate XopL-mediated cell death in $\mathrm{Nb}$ and the potential role of $\mathrm{NbFd}$, we designed construct 


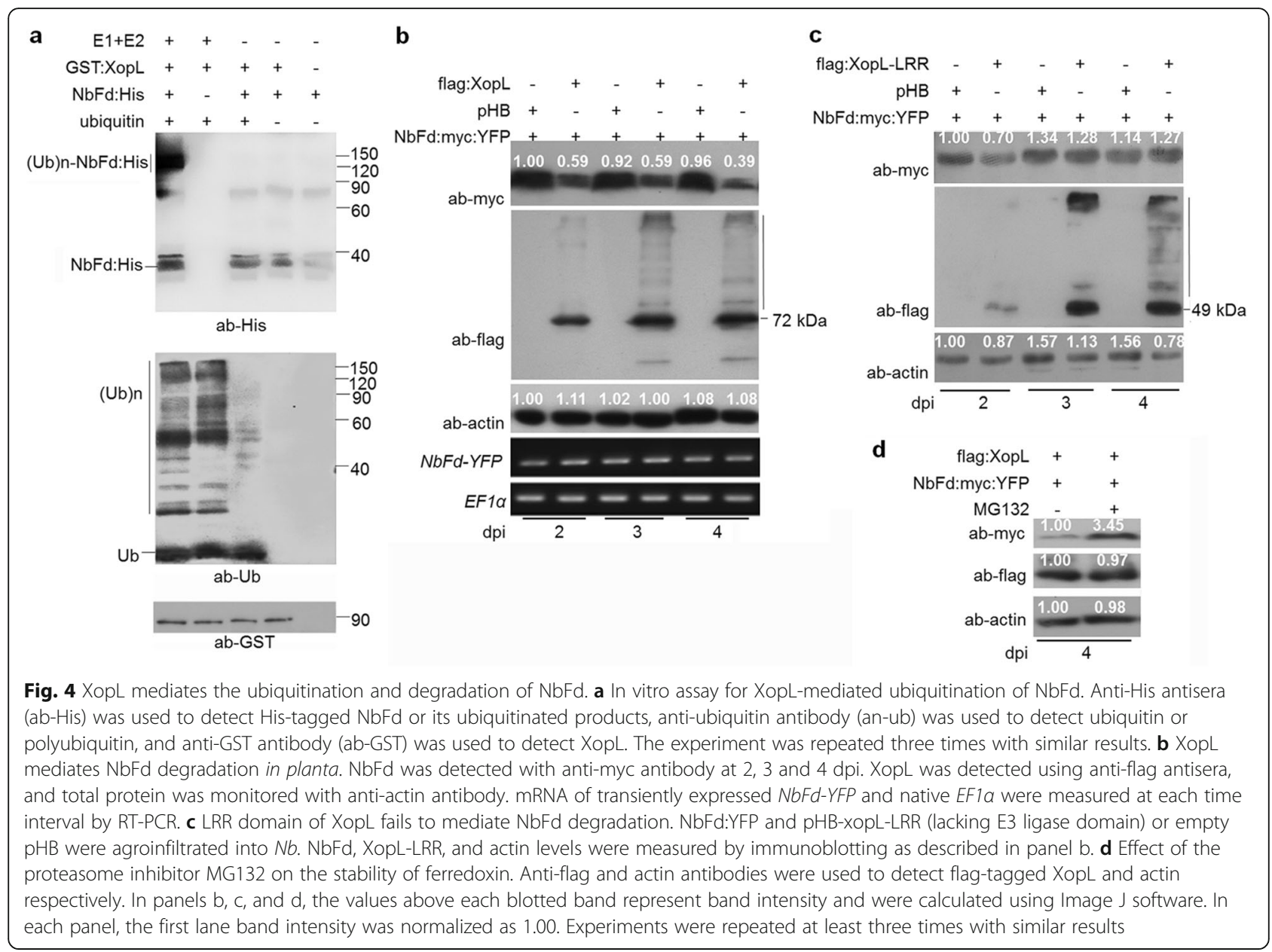

pCA- $2 \times \mathrm{NbFd}$ for RNAi-mediated silencing of $\mathrm{NbFd}$ (Fig. 5a). DNA sequence blasting against the $\mathrm{Nb}$ database housed in SolGenomics (https://solgenomics.net) revealed $4 \mathrm{NbFd-encoding}$ genes sharing high identity (98-99\%). The region of NbFd selected for silencing contained $33 \mathrm{bp}$ of the chloroplast peptide and $368 \mathrm{bp}$ of the functional domain (Fig. 3a) and was able to match all of the four NbFd coding genes. The $N b F d$-silenced plant lines showed a dramatic decrease in $\mathrm{NbFd}$ expression (approximately $3 \%$ of EV1300 lines, transformed with empty vector) in the $\mathrm{T}_{1}$ generation (Fig. $5 \mathrm{~b}$ ). Furthermore, the silencing of $\mathrm{NbFd}$ resulted in mottled, chlorotic leaves (Fig. 5c). Ferredoxin is an ion carrier in chloroplasts and plays an important role in maintaining redox homeostasis in the organelle (Hanke et al. 2004); thus a sharp decrease in ferredoxin levels could impact the redox state of cells and potentially cause ROS accumulation. To investigate this further, we examined $\mathrm{H}_{2} \mathrm{O}_{2}$ accumulation in $\mathrm{NbFd}$-si-

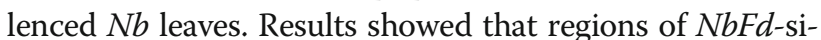
lenced leaves were stained brown (Fig. 5e) indicating an accumulation of ROS. Whereas, this staining did not occur in EV1300 leaves.
Next, we evaluated the induction of cell death in $N b F d$-silenced plants. At $3 \mathrm{dpi}$, both XopL and $\mathrm{pHB}$ induced cell death in $N b F d$-silenced leaves; however, no cell death was observed in leaves of EV1300 infiltrated with $\mathrm{pHB}$ in the inoculated region at $7 \mathrm{dpi}$ (Fig. 5d). These results suggest that the silencing of $N b F d$ in $\mathrm{Nb}$ renders cell death, which was apparent when both $\mathrm{pHB}$ and $\mathrm{pHB}$-xopL were introduced.

\section{ROS accumulation is a prerequisite for XopL-induced cell death}

Given that XopL mediated the degradation of NbFd (Fig. 4b) and induced ROS accumulation (Fig. 2d), we speculated that ROS accumulation may be important for XopLmediated cell death in $N b$. In order to investigate this, we co-infiltrated pHB-XopL with NAC (N-acetyl-L-cysteine), which was used as an ROS scavenger (Valassakis et al. 2018). NAC inhibited both ROS accumulation (Fig. 6a) and XopL-mediated cell death (Fig. 6b). These results indicate the vital role of ROS accumulation in XopLinduced cell death. 


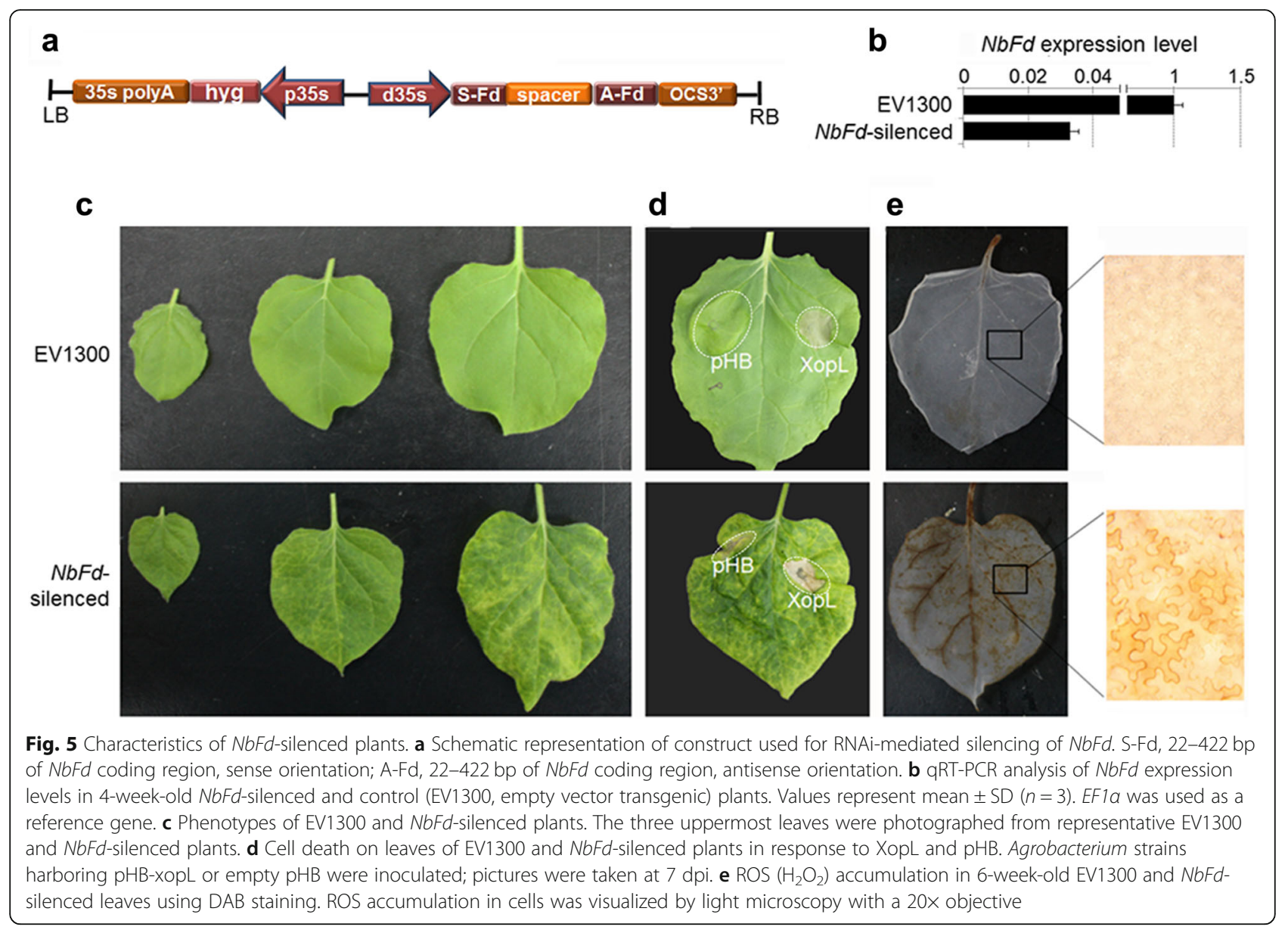

\section{Discussion}

Ferredoxins comprise a group of small [2Fe-2S]-containing proteins that are encoded in the nucleus. Fds are synthesized in the cytosol as precursors and are translocated across the chloroplast envelope via an $\mathrm{N}$-terminal extension called the signal (transit) peptide (Pilon et al.
1992; Holbrook et al. 2016). In the current study, NbFd: YFP was observed in both chloroplasts and cytosol (Additional file 3: Figure S2), which is consistent with the intracellular transport process of the $\mathrm{Fd}$ pre-protein (Pilon et al. 1992). The interaction between NbFd and XopL is in cytoplasm (Fig. 3d), which suggests that
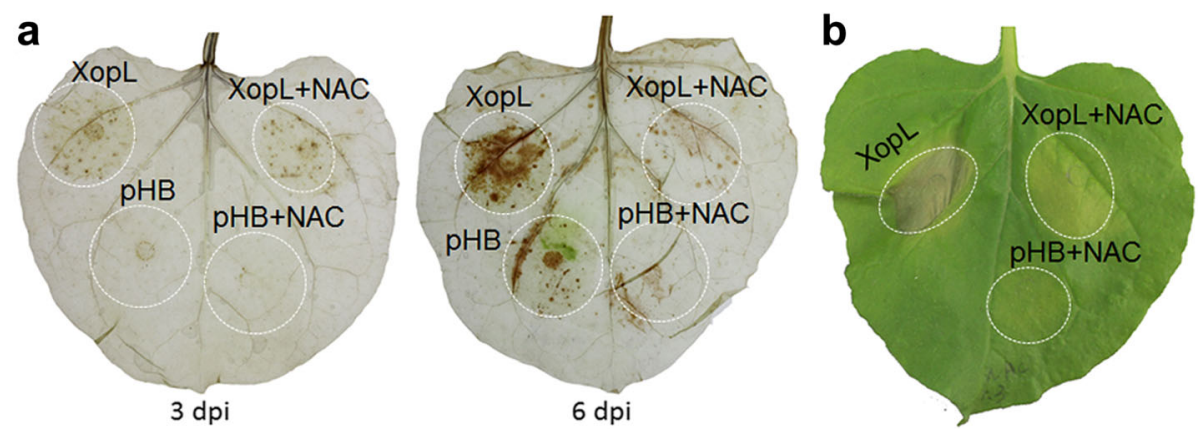

Fig. 6 Effect of the ROS scavenger N-acetyl-L-cysteine (NAC) on XopL-induced cell death. Agrobacterium strains harboring pHB-xopL or empty $\mathrm{pHB}$ were inoculated into the wild-type $\mathrm{Nb}$ leaves at $\mathrm{OD}_{600}=1.0$; $\mathrm{NAC}$ was co-infiltrated at a concentration of $2 \mathrm{mM}$. $\mathbf{a}$ Effect of NAC on XopLinduced ROS accumulation at 3 and 6 dpi. Agrobacterium-infected Nb leaves were collected at 3 and 6 dpi and stained in DAB solution. b Phenotypes of Nb leaves infiltrated with pHB-XopL and pHB, with or without NAC. Pictures were taken at $7 \mathrm{dpi}$. Experiments were repeated three times with similar results 
XopL-induced defense related response may be due to NbFd imbalance in chloroplast. Depletion of NbFd as an electron acceptor would enhance production of ROS and ion leakage of cells (Lintala et al. 2012). Chloroplast-generated ROS have been closely associated with cell death (Liu et al. 2007; Zurbriggen et al. 2009) and serve as messengers to initiate the expression of defense genes (Shapiguzov et al. 2012). The C-terminus of $\mathrm{NbFd}(\mathrm{NbFd} \Delta 2-51)$ contains the iron-sulfur site and cysteine residues that catalyze electron transport from photosystem I to acceptors located in the chloroplast stroma (Hanke and Mulo 2013). Although lacking the signal peptide for translocation into the chloroplast, $\mathrm{NbFd} \Delta 2-51$ interacted with XopL in yeast two-hybrid and BiFC assays (Fig. 3b, d). We did not evaluate whether XopL interacts with the signal peptide of NbFd, so it remains unclear whether they interact with each other.

Ubiquitination is a posttranslational modification involving the covalent addition of a single ubiquitin molecule or a polyubiquitin chain onto a lysine residue of the target protein (Kerscher et al. 2006). In this study, we show that $\mathrm{XopL}_{X o o}$ ubiquitinates $\mathrm{NbFd}$, which leads to the degradation of $\mathrm{NbFd}$ via the ubiquitin-proteasome system (Fig. 4b-d). There are seven lysine residues distributed in $\mathrm{NbFd}$, with four in the signal peptide and three in the $\mathrm{C}$-terminus. However, the exact lysine site(s) in $\mathrm{NbFd}$ for the ubiquitination needs further investigation.

Our data suggest that the C-terminal end of XopL is required since XopL-LRR failed to catalyze the degradation of $\mathrm{NbFd}$ (Fig. 4c). In the degradation assays, multiple immuno-reactive XopL and XopL-LRR bands were observed at later time points (Fig. 4b, c), suggesting that XopL is modified at the $\mathrm{N}$-terminus when transiently expressed in $N b$. The potential modification is ubiquitination but not auto-ubiquitination of the effector, because the E3 ligase deleted mutant (Xop-LRR) of XopL was also modified (Fig. 4c). Although autoubiquitination has been reported for multiple effectors in the IpaH family (Rohde et al. 2007), including NopM in Sinorhizobium fredii (Xu et al. 2018), the effort to show GST:XOPL self-ubiquitination in vitro was unsuccessful (Additional file 7: Figure S5), which indicates that XopL might not be able to ubiquitinate itself.

Stromules (stroma-filled tubules that emanate from plastid bodies) are highly abundant during ETI in $\mathrm{Nb}$ and are potentially involved in the transport of defense signals from the chloroplast to the nucleus (Caplan et al. 2015). Recently, it was reported the expression of $\mathrm{XopL}_{X c v}$ in $\mathrm{Nb}$ resulted in massive changes in cell morphology including cells that were almost completely devoid of stromules. In contrast, a mutant of XopL $\mathrm{X}_{\overline{ }}$ containing the LRR domain but lacking E3 ligase activity failed to eliminate stromules and was localized to microtubules (Erickson et al. 2018). The authors speculated that the XopL mutant likely binds potential XopL targets through the LRR domain, but is unable to ubiquitinate them. In our work, we observed that XopL-LRR interacted with NbFd in the yeast twohybrid and BiFC assays (Fig. 3); this supports a model in which the LRR domain of XopL interacts with NbFd. Additionally, $F d 2$, a foliar ferredoxin in Arabidopsis, was found to be localized to stromules when transiently expressed in $N b$. The $F d 2$ knock-out mutant showed compromised defense and reduced ROS accumulation, indicating a role for $\mathrm{Fd} 2$ in the plant immune response (Wang et al. 2018). The authors suggested that Fd2 might function in retrograde signal transduction from the chloroplast to the nuclear envelope via stromules. Although stromules were not evaluated in our study, it remains plausible that the XopL-mediated degradation of NbFd may cause changes of stromules.

Singer et al. hypothesize that XopL has multiple roles including the suppression of PTI via its LRR region, the ubiquitination of a plant substrate that may be recognized by the LRR region, and the elicitation of programmed cell death that requires both the LRR region and XL-box of XopL (Singer et al. 2013). Our yeast twohybrid results indicate that NbFd interacts with both the LRR- and XL-box-containing domains of $\mathrm{XopL}_{X o o}$, but these two separate domains have lower binding abilities than the full length XopL towards NbFd (Fig. 3b). Thus we speculate that NbFd binds to XopL at the junction of LRR and XL-box with two binding sites. The interaction of XopL with NbFd and subsequent ubiquitination of $\mathrm{NbFd}$ is an interesting and novel finding, but the evolutionary forces resulting in the ubiquitination of NbFd by a Xanthomonas effector remain a mystery. Although ubiquitin and ferredoxin have no obvious analogy in function or conserved sequence motifs, it is important to note that structural similarities between these two proteins have been reported previously (Michnick and Shakhnovich 1998). Ferredoxin has topological features and dimensions of structural elements that are conserved in ubiquitin, and the two proteins share a conserved folding motif known as the $\beta$-grasp (Vriend and Sander 1991; Fukuyama 2004). It is interesting to speculate that ubiquitin and ferredoxin may have a common ancestor and perhaps their shared topology might help explain how XopL evolved to recognize and interact with these two proteins.

\section{Conclusions}

In the study we report the cell death-inducing ability of four type III secretion system effectors of $X$. oryzae in $N b$. Specially, one of the effectors, XopL, holding LRR region at its $\mathrm{N}$-terminus and E3 ubiquitin ligase domain at the C-terminus, interacts with $\mathrm{Nb}$ ferredoxin $(\mathrm{NbFd})$. 
The interaction results in the ubiquitination and degradation of NbFd and subsequently leads to ROS production and defense-related response in plants. Taken together, our findings enlarge the repertoire of effector targets to include key components of plant defense systems.

\section{Methods}

\section{Bacterial strains, plasmid and plant materials}

The bacterial strains and plasmids used in this study are listed in Additional file 4: Table S2. Escherichia coli strains were cultivated in Luria-Bertani (LB) medium at $37^{\circ} \mathrm{C}$ (Sambrook and Russell 2001). X. oryzae pv. oryzae PXO99 ${ }^{\mathrm{A}}$ and $\mathrm{X}$. oryzae pv. oryzicola RS105 were cultivated in nutrient broth (NB) or NB with agar at $28^{\circ} \mathrm{C}$ (Li et al. 2011). Agrobacterium was grown in LB medium containing rifampicin at $28^{\circ} \mathrm{C}$. Saccharomyces cerevisiae was grown in Yeast Extract Peptone Dextrose medium at $28^{\circ} \mathrm{C}$. Antibiotics were used at the following final concentrations: ampicillin, $100 \mu \mathrm{g} / \mathrm{mL}$; rifampicin, $75 \mu \mathrm{g} / \mathrm{mL}$; and kanamycin, $25 \mu \mathrm{g} / \mathrm{mL}$.

$\mathrm{Nb}$ plants were cultivated at $25^{\circ} \mathrm{C}$ in a growth chamber with a $16 \mathrm{~h}$ light $/ 8 \mathrm{~h}$ dark photoperiod.

\section{Detection of cell death}

Six-week old leaves were inoculated for transient expression of effectors as described above. At $7 \mathrm{dpi}$, the inoculated leaves were collected and washed in water, then submerged in a $0.25 \%(\mathrm{w} / \mathrm{v})$ aqueous solution of Evans blue for $24 \mathrm{~h}$ at room temperature. The stained leaves were washed three times ( $15 \mathrm{~min} /$ wash) with gentle agitation in PBS plus Tween $(0.05 \%, \mathrm{v} / \mathrm{v})$ to remove excess dye (Wright et al. 2000). Leaves were then transferred to 95\% ethanol in a boiling water bath for bleaching until chlorophyll was completely removed. Samples were stored and examined in 65\% ethanol and photographed under white light. Three independent plants were used, and experiments were repeated three times with similar results.

\section{DAB staining for ROS accumulation}

In situ $\mathrm{H}_{2} \mathrm{O}_{2}$ accumulation was detected using 3, 3' -diaminobenzidine (DAB) staining as described previously (Bindschedler et al. 2006) with minor modifications. Briefly, Agrobacterium-infected six-week old $N b$ leaves were collected at different time points and submerged in a DAB $(1 \mathrm{mg} / \mathrm{mL})$ solution containing $0.05 \%$ Tween$20(\mathrm{v} / \mathrm{v})$ and $10 \mathrm{mM}$ sodium phosphate buffer ( $\mathrm{pH} 7.0)$ under darkness for $8 \mathrm{~h}$. Leaves were carefully transferred to $95 \%$ ethanol and placed in a boiling water bath until chlorophyll was completely removed. Samples were stored in 65\% ethanol and then photographed under white light. N-acetyl-L-cysteine (NAC; $2 \mathrm{mM}$ ) was used as a ROS scavenger and co-infiltrated with
Agrobacterium. Three independent plants were used, and experiments were repeated three times.

\section{Yeast two-hybrid assays}

The Matchmaker ${ }^{\text {Tx }}$ GAL4 two-hybrid system (Clontech, CA) was used to screen for XopL-interacting proteins in this study. The xopL coding sequence in pGBKT7-xopL was used to generate bait for screening a $\mathrm{Nb}$ cDNA library of mature leaves. SD medium was used for screening assays. $1 \times$ Amino Acid Dropout Mix (Clontech, CA) was added to SD, including SD/-Trp-Leu-His (lacking tryptophan, leucine, and histidine) and SD/ -Trp-Leu-His-Ade (lacking tryptophan, leucine, histidine and adenine).

The yeast strain AH109 was co-transformed with pGBKT7-xopL and the $\mathrm{Nb}$ cDNA library and cultured on SD/-Leu-Trp-His medium. Transformants were then transferred to $\mathrm{SD} /$-Leu-Trp-His-Ade agar medium containing $20 \mathrm{mg} / \mathrm{mL} \mathrm{X- \alpha -GAL}$. Blue colonies were considered to be potential positive interactions; positives were amplified using primer pair T7/3AD (Additional file 8: Table S3), and PCR products were sequenced.

Interactions of XopL, $\mathrm{NbFd}$ and their derivatives were analyzed on SD/-Leu-Trp-His-Ade/X- $\alpha$-GAL medium. Briefly, transformants were grown on SD/-Leu-Trp-His medium and then transferred to SD/-Leu-Trp-His-Ade agar medium. Single colonies from SD/-Leu-Trp-HisAde agar medium were picked and inoculated into $1 \mathrm{~mL}$ $\mathrm{SD} /$-Leu-Trp-His-Ade liquid medium with shaking for $24 \mathrm{~h}$. Yeast cells were harvested by centrifugation $(5000$ $\mathrm{rpm}, 2 \mathrm{~min})$ and resuspended in sterilized deionized water $\left(\mathrm{OD}_{600}=0.5\right)$. The water-suspended yeast was 10 fold serial diluted and $10 \mu \mathrm{L}$ was spotted on SD/-LeuTrp-His-Ade agar medium with and without $20 \mathrm{mg} / \mathrm{mL}$ $\mathrm{X}-\alpha-\mathrm{GAL}$.

\section{Co-immunoprecipitation (ColP) assay}

$3 \times$ flag: XopL was co-expressed with NbFd:YFP or YFP in $\mathrm{Nb}$ leaves by introducing pHB-xopL, pNbFd-YFP and pYFP (Additional file 4: Table S2) into $\mathrm{Nb}$. At 48 h postinfiltration (hpi), total proteins in the infiltrated region were extracted with lysis buffer $[50 \mathrm{mM}$ Tris- $\mathrm{HCl}(\mathrm{pH}$ 7.5), $150 \mathrm{mM} \mathrm{NaCl}, 1 \mathrm{mM}$ EDTA, $10 \%$ glycerol, $0.2 \%$ Triton-X 100, $1 \mathrm{mM}$ PMSF and proteinase inhibitor cocktail). For preclearing, the extracted proteins were mixed with $30 \mu \mathrm{L}$ protein A magnetic beads (Bimake, USA) for $15 \mathrm{~min}$ at $4{ }^{\circ} \mathrm{C}$. After removing the beads, the precleared total proteins were incubated with $2.5 \mu \mathrm{g}$ anti-myc antibody (TransGen Biotech, China) and $30 \mu \mathrm{L}$ protein A magnetic beads for $10 \mathrm{~h}$ at $4{ }^{\circ} \mathrm{C}$. The beads were washed five times with washing buffer (Tris- $\mathrm{HCl}$ pH 7.5, $300 \mathrm{mM} \mathrm{NaCl}, 1 \mathrm{mM}$ EDTA, 10\% glycerol, $1 \%$ Triton-X 100). The beads were suspended in loading buffer, boiled for $8 \mathrm{~min}$, and the supernatant was used in 
immunoblotting assays. Proteins were separated in 10\% SDS-PAGE gels and transferred to PVDF membranes $(0.2 \mu \mathrm{m})$. Anti-flag and anti-myc antibodies (TransGen Biotech, China) were used for detecting $3 \times$ flag: XopL and YFP fusions, respectively.

\section{Localization and bimolecular fluorescence complementation (BiFC) assays}

BiFC assays were performed as described previously (Walter et al. 2004) with minor modifications. Buffersupplemented Agrobacterium strains containing pXopL-YN and $\mathrm{pNbFd-YC}$ were mixed (1:1 ratio) and incubated at $25^{\circ} \mathrm{C}$ for $1 \mathrm{~h}$. Controls included cotransformation with XopL:YN and empty YC vector and NbFd:YC (Fd:YC) with empty $\mathrm{YN}$ vector. Fluorescence from infiltrated $\mathrm{Nb}$ leaves was monitored and imaged at 48 hpi with a confocal laser fluorescence microscope Leica TCS SP5-II Confocal Microscope (Leica Microsystems, Wetzlar, Germany). Chloroplasts were visualized by auto-fluorescence.

\section{In vitro ubiquitination assay}

To evaluate the potential ubiquitination of NbFd by XopL, we used the Ubiquitin Conjugation Kit K-960 (Boston Biochem, Cambridge, MA, USA). Ubiquitin aldehyde (Boston Biochem) was used to inhibit deubiquitinating enzymes. $10 \mu \mathrm{L}$ conjugation fraction $\mathrm{A}, 5 \mu \mathrm{M}$ GST:XopL, $1.7 \mu \mathrm{L}$ ubiquitin solution and $1 \mu \mathrm{L}$ ubiquitin aldehyde were gently mixed and incubated for $5 \mathrm{~min}$ at $37^{\circ} \mathrm{C}$. Next, $2.5 \mu \mathrm{L}$ energy solution and $2 \mu \mathrm{g} \mathrm{NbFd:His}{ }_{6}$ were added, gently mixed, and incubated for $4 \mathrm{~h}$ at $37^{\circ} \mathrm{C}$. The reaction was terminated with $10 \mathrm{mM}$ DTT; $5 \times$ loading buffer was added and the reactions were boiled for $8 \mathrm{~min}$. The samples were separated by $10 \%$ SDS-PAGE, transferred to PVDF membranes $(0.2 \mu \mathrm{m})$ and probed with anti-His antibody (Abgent, San Diego, USA). After washing with stripping buffer (glycine 1.88 $\mathrm{g} / \mathrm{L}$, SDS $20 \mathrm{~g} / \mathrm{L}, \mathrm{pH} 2.0$ ), the membrane was re-probed with anti-GST antibody (TransGen Biotech, China). After a final stripping step, the membrane was probed a third time using ubiquitin antibody (Abmart, China).

\section{NbFd degradation assay}

To detect the potential degradation of NbFd by XopL and XopL-LRR in planta, pHB-xopL or pHB-XopL-LRR were co-infiltrated with pNbFd-YFP into $\mathrm{Nb}$ leaves at a $3: 2$ ratio. At 2, 3, and $4 \mathrm{dpi}$, total proteins were extracted with lysis buffer (described above in the CoIP assay). Protein concentrations were determined using the Bradford Coomassie blue method and adjusted to be identical. Total proteins $(100 \mu \mathrm{L})$ were mixed with $5 \times$ SDS loading buffer and boiled for $8 \mathrm{~min}$. Proteins were separated by $10 \%$ SDS-PAGE and then transferred to PVDF membranes $(0.2 \mu \mathrm{m})$. For initial immunoblotting, an anti-myc antibody was used to detect NbFd:YFP and visualized by chemiluminescence. The antibody complex was then removed with stripping buffer, blocked and probed with anti-flag antisera to detect XopL. The membrane was stripped a final time and incubated with anti-actin antibody to detect the total protein level. The role of the ubiquitin proteasome system in degradation of NbFd was investigated by introducing proteasome inhibitor MG132 $(100 \mu \mathrm{M})$ into the agroinfiltrated leaves $16 \mathrm{~h}$ before samples were harvested.

\section{Supplemental methods}

See Additional file 9.

\section{Supplementary information}

Supplementary information accompanies this paper at https://doi.org/10. 1186/s42483-020-00055-w. Additional file 1: Table S1. Primers used for amplifying genes
encoding T3E from X. oryzae pv. oryzicola.

Additional file 2: Figure S1. Ion leakage measurements. Quantification of cell death using ion leakage measurements in $\mathrm{Nb}$ expressing XopL or empty vector (pHB) at 3 and $7 \mathrm{dpi}$. Values represent mean conductivity \pm SD $(n=3)$. Asterisks indicate significant differences (Student's $t$ test, $P<$ 0.05 ) relative to the empty vector $\mathrm{pHB}$. Experiment was repeated three times with similar results.

Additional file 3: Figure S2. Localization of XopL:YFP and NdFd:YFP fusions. a Subcellular localization assay of XopL:YFP and NbFd:YFP (Fd:YFP) fusions in Nb. YFP was expressed as control. Images were obtained with a Leica confocal microscope. The excitation wavelength for YFP was $488 \mathrm{~nm}$, and 520-550 nm was used for emission. Chloroplasts were visualized by auto-fluorescence. Blue arrows and red arrows indicate localization of ferredoxin in chloroplasts. Bars represent $20 \mu \mathrm{m}$. b Expression of each YFP fused protein were detected by Western blot using antiGFP antibody. ${ }^{*}$ in the first lane indicates non-specific band.

Additional file 4: Table S2. Strains and plasmids used in this study. Additional file 5: Figure S3. Analysis of XopL and NbFd interaction by yeast two-hybrid assays. XopL and $\mathrm{Nb}$ ferredoxin ( $\mathrm{NbFd}$ ) were cloned into pGADT7 and pGBKT7, respectively. Yeast liquid broth was diluted at original $\mathrm{OD}_{600}$ value of 0.5 and spotted on SD/-Leu-Trp-His-Ade agar medium with or without X-a-gal. The experiments were performed three times with similar results.

Additional file 6: Figure S4. XopL sequence alignment and structure. a Alignment of $X_{o p} L_{X a p}, X_{o p} L_{X_{o o}}$ and $X_{o p} L_{X_{c v}}$ from $X$. axonopodis pv. punicae strain ITCCBD0003, Xoo PXO99 ${ }^{A}$, and $X$. campestris pv. vesicatoria 85-10 (GenBank accession no. CAJ24951), respectively. DNAMAN (Lynnon Biosoft, San Ramon, CA, USA) was used to construct the alignment. Identical amino acids are indicated in lower case. Identical amino acids were highlighted with dark blue, and amino acids shared by any two proteins were highlighted with light blue. Blue arrows indicate $\beta$-sheets; red rectangles indicate $a$-helices. Helical regions $<5$ residues are marked and not labeled. $\mathbf{b}$ Ribbon diagram of the N-terminal LRR domain of XopL [aa 144-436]. c Ribbon diagram of the C-terminal XL-box (E3 ligase

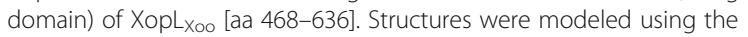
Swiss Model algorithm (www.swissmodel.expasy.org/); $\beta$-sheets and ahelices are labeled. $\mathbf{d}$ Electrostatic surface of the XopL-C terminus [aa 468-636] showing the net negative charge.

Additional file 7: Figure S5. Uncropped western blot picture in Fig. 4a bottom panel. The reactions for in vitro ubiquitination assay were immune blotted using anti-GST antibody (ab-GST) to detect GST:XopL.

Additional file 8: Table S3. Primers used in this study.

Additional file 9: Supplemental methods. Methods S1, DNA manipulation and plasmid construction. Methods S2, transient expression in Nb. Methods S3, protein production and purification. Methods S4, RNA 
isolation and gene expression analysis. Method S5, ion leakage test. Methods S6, overexpression and RNAi-mediated silencing of NbFd.

\section{Abbreviations}

BB: Bacterial blight; BLS: Bacterial leaf streak; DAB: 3, 3'-diaminobenzidine; dpi: Days post infiltration; ETI: Effector-triggered immunity; EV1300: Empty vector pCAMBIA1300 transgenic Nb plant; HR: Hypersensitive response; LRR: Leucine-rich repeats; NAC: N-acetyl-L-cysteine; Nb: Nicotiana benthamiana; NbFd: Nicotiana benthamiana ferredoxin; NHR: Nonhost resistance; PAMPs: Pathogen-associated molecular patterns; PRRs: Patternrecognition receptors; PTI: PAMP-triggered immunity; ROS: Reactive oxygen species; T3E: T3SS effector; T3SS: Type III secretion system; TALE: Transcription activator-like effectors; Xcv: Xanthomonas campestris pv. vesicatoria; Xoc: Xanthomonas oryzae pv. oryzicola; Xoo: Xanthomonas oryzae pv. oryzae; Xop: Xanthomonas outer protein

\section{Authors' contributions}

WM and GC designed the experiments; WM performed the experiments; LZ, $L C, Y C$ and BZ contributed materials; XX provided assistance for protein purification and western blot; FH contributed constructions; WM and GC wrote the paper; JA revised the manuscript. All authors read and approved the final manuscript.

\section{Funding}

This study was supported by the National Natural Science Foundation of China (31830072) and the National Transgenic Major Program (2016ZX08001-002).

\section{Availability of data and materials}

The datasets used during the current study are available from the corresponding author on reasonable request.

\section{Ethics approval and consent to participate} Not applicable.

\section{Consent for publication}

Not applicable.

\section{Competing interests}

The authors declare that they have no competing interests.

\section{Author details}

${ }^{1}$ School of Agriculture and Biology/State Key Laboratory of Microbial Metabolism, Shanghai Jiao Tong University, Shanghai 200240, China. ${ }^{2}$ Current working address: Citrus Research and Education Center, Institute of Food and Agriculture Sciences, University of Florida, Lake Alfred, FL 33850, USA. ${ }^{3}$ Current working address: Department of Plant Pathology, University of Florida, Gainesville, FL 32611, USA. ${ }^{4}$ The Center for Plant Science Innovation, University of Nebraska, Lincoln, NE 68588-0660, U.S.A.

\section{Received: 12 April 2020 Accepted: 28 May 2020}

\section{Published online: 24 June 2020}

\section{References}

Adlung N, Prochaska H, Thieme S, Banik A, Bluher D, John P, et al. Non-host resistance induced by the Xanthomonas effector XopQ is widespread within the genus Nicotiana and functionally depends on EDS1. Front Plant Sci. 2016; 7:1796.

Bindschedler LV, Dewdney J, Blee KA, Stone JM, Asai T, Plotnikov J, et al. Peroxidase-dependent apoplastic oxidative burst in Arabidopsis required for pathogen resistance. Plant J. 2006;47:851-63.

Blüher D, Laha D, Thieme S, Hofer A, Eschen-Lippold L, Masch A, et al. A 1phytase type III effector interferes with plant hormone signaling. Nat Commun. 2017:8:2159.

Büttner D. Behind the lines-actions of bacterial type III effector proteins in plant cells. FEMS Microbiol Rev. 2016;40:894-937.

Caplan JL, Kumar AS, Park E, Padmanabhan MS, Hoban K, Modla S, et al. Chloroplast stromules function during innate immunity. Dev Cell. 2015;34:45-57.

Sambrook J, Russell DW. Molecular cloning: A laboratory manual (3rd edition) New York: Cold Spring Harbor Laboratory Press; 2001.
Dean P. Functional domains and motifs of bacterial type III effector proteins and their roles in infection. FEMS Microbiol Rev. 2011:35:1100-25.

Dodds PN, Rathjen JP. Plant immunity: towards an integrated view of plantpathogen interactions. Nat Rev Genet. 2010;11:539-48.

Erickson JL, Adlung N, Lampe C, Bonas U, Schattat MH. The Xanthomonas effector XopL uncovers the role of microtubules in stromule extension and dynamics in Nicotiana benthamiana. Plant J. 2018;93:856-70.

Fan J, Doerner P. Genetic and molecular basis of nonhost disease resistance: complex, yes; silver bullet, no. Curr Opin Plant Biol. 2012;15:400-6.

Fukuyama K. Structure and function of plant-type ferredoxins. Photosynth Res. 2004;81:289-301.

Gopalan S, Wei W, He SY. hrp gene-dependent induction of hin1: a plant gene activated rapidly by both harpins and the avrPto gene-mediated signal. Plant J. 1996;10:591-600.

Hanke G, Mulo P. Plant type ferredoxins and ferredoxin-dependent metabolism Plant Cell Environ. 2013;36:1071-84.

Hanke GT, Kimata-Ariga Y, Taniguchi I, Hase T. A post genomic characterization of Arabidopsis ferredoxins. Plant Physiol. 2004;134:255-64.

Holbrook K, Subramanian C, Chotewutmontri P. Reddick LE, Wright S, Zhang $\mathrm{H}_{4}$ et al. Functional analysis of semi-conserved transit peptide motifs and mechanistic implications in precursor targeting and recognition. Mol Plant. 2016;9:1286-301

Ishikawa K, Yamaguchi K, Sakamoto K, Yoshimura S, Inoue K, Tsuge S, et al. Bacterial effector modulation of host E3 ligase activity suppresses PAMPtriggered immunity in rice. Nat Commun. 2014;5:5430.

Jacques MA, Arlat M, Boulanger A, Boureau T, Carrere S, Cesbron S, et al. Using ecology, physiology, and genomics to understand host specificity in Xanthomonas. Annu Rev Phytopathol. 2016;54:163-87.

Kay S, Bonas U. How Xanthomonas type III effectors manipulate the host plant. Curr Opin Microbiol. 2009:12:37-43.

Kerscher O, Felberbaum R, Hochstrasser M. Modification of proteins by ubiquitin and ubiquitin-like proteins. Annu Rev Cell Dev Biol. 2006;22:159-80.

Khan M, Subramaniam R, Desveaux D. Of guards, decoys, baits and traps: pathogen perception in plants by type III effector sensors. Curr Opin Microbiol. 2016;29:49-55.

Lacombe S, Rougon-Cardoso A, Sherwood E, Peeters N, Dahlbeck D, van Esse HP et al. Interfamily transfer of a plant pattern-recognition receptor confers broad-spectrum bacterial resistance. Nat Biotechnol. 2010;28:365-9.

Lee AH-Y, Middleton MA, Guttman DS, Desveaux D. Phytopathogen type III effectors as probes of biological systems. Microb Biotechnol. 2013;6:230-40.

Li YR, Zou HS, Che YZ, Cui YP, Guo W, Zou LF, et al. A novel regulatory role of HrpD6 in regulating hrp-hrc-hpa genes in Xanthomonas oryzae pv. oryzicola. Mol Plant-Microbe Interact 2011:24:1086-101.

Lin YH, Machner MP. Exploitation of the host cell ubiquitin machinery by microbial effector proteins. J Cell Sci. 2017;130:1985-96.

Lintala M, Lehtimaki N, Benz JP, Jungfer A, Soll J, Aro EM, et al. Depletion of leaf-type ferredoxin-NADP(+) oxidoreductase results in the permanent induction of photoprotective mechanisms in Arabidopsis chloroplasts. Plant J. 2012;70:809-17.

Liu Y, Ren D, Pike S, Pallardy S, Gassmann W, Zhang S. Chloroplast-generated reactive oxygen species are involved in hypersensitive response-like cell death mediated by a mitogen-activated protein kinase cascade. Plant J. 2007;51:941-54.

Michnick SW, Shakhnovich E. A strategy for detecting the conservation of folding-nucleus residues in protein superfamilies. Fold Des. 1998;3:239-51.

Mysore KS, Ryu CM. Nonhost resistance: how much do we know? Trends Plant Sci. 2004;9:97-104.

Nguyen HP, Chakravarthy S, Velasquez AC, McLane HL, Zeng L, Nakayashiki H, et al. Methods to study PAMP-triggered immunity using tomato and Nicotiana benthamiana. Mol Plant-Microbe Interact. 2010;23:991-9.

Nino-Liu DO, Ronald PC, Bogdanove AJ. Xanthomonas oryzae pathovars: model pathogens of a model crop. Mol Plant Pathol. 2006;7:303-24.

Pilon M, de Kruijff B, Weisbeek PJ. New insights into the import mechanism of the ferredoxin precursor into chloroplasts. J Biol Chem. 1992;267:2548-56

Popov G, Majhi BB, Sessa G. Effector gene xopAE of Xanthomonas euvesicatoria 85-10 is part of an operon and encodes an E3 ubiquitin ligase. J Bacteriol. 2018;200:e00104-18.

Pruneda JN, Durkin CH, Geurink PP, Ovaa H, Santhanam B, Holden DW, et al. The molecular basis for ubiquitin and ubiquitin-like specificities in bacterial effector proteases. Mol Cell. 2016;63:261-76.

Qin J, Zhou X, Sun L, Wang K, Yang F, Liao H, et al. The Xanthomonas effector XopK harbours E3 ubiquitin-ligase activity that is required for virulence. New Phytol. 2018;220:219-31. 
Rohde JR, Breitkreutz A, Chenal A, Sansonetti PJ, Parsot C. Type III secretion effectors of the IpaH family are E3 ubiquitin ligases. Cell Host Microbe. 2007; 1:77-83.

Schornack S, Moscou MJ, Ward ER, Horvath DM. Engineering plant disease resistance based on TAL effectors. Annu Rev Phytopathol. 2013;51:383-406.

Schultink A, Qi T, Lee A, Steinbrenner AD, Staskawicz B. Roq1 mediates recognition of the Xanthomonas and Pseudomonas effector proteins XopQ and HopQ1. Plant J. 2017;92:787-95.

Schwartz AR, Potnis N, Timilsina S, Wilson M, Patane J, Martins J Jr, et al. Phylogenomics of Xanthomonas field strains infecting pepper and tomato reveals diversity in effector repertoires and identifies determinants of host specificity. Front Microbiol. 2015;6:535.

Schwessinger B, Ronald PC. Plant innate immunity: perception of conserved microbial signatures. Annu Rev Plant Biol. 2012;63:451-82.

Senthil-Kumar M, Mysore KS. Nonhost resistance against bacterial pathogens: retrospectives and prospects. Annu Rev Phytopathol. 2013;51:407-27.

Shapiguzov A, Vainonen JP, Wrzaczek M, Kangasjarvi J. ROS-talk-how the apoplast, the chloroplast, and the nucleus get the message through. Front Plant Sci. 2012;3:292.

Singer AU, Schulze S, Skarina T, Xu X, Cui H, Eschen-Lippold L, et al. A pathogen type III effector with a novel E3 ubiquitin ligase architecture. PLoS Pathog. 2013;9:e1003121.

Stork W, Kim JG, Mudgett MB. Functional analysis of plant defense suppression and activation by the Xanthomonas core type III effector XopX. Mol PlantMicrobe Interact. 2015;28:180-94.

Sun YJ, Ma WX, Cai LL, Liu L, Zou LF, Chen GY. Identification of a protein both in tobacco and rice that interacts with an HR-elicitor SsbX of Xanthomonas oryzae pv. oryzicola. Sci Agric Sin. 2015;48(4):683-94 (in Chinese). https://doi. org/10.3864/j.issn.0578-1752.2015.04.06.

Thieme F, Szczesny R, Urban A, Kirchner O, Hause G, Bonas U. New type III effectors from Xanthomonas campestris pv. vesicatoria trigger plant reactions dependent on a conserved N-myristoylation motif. Mol Plant-Microbe Interact. 2007;20:1250-61.

Uma B, Rani TS, Podile AR. Warriors at the gate that never sleep: non-host resistance in plants. J Plant Physiol. 2011;168:2141-52.

Ustun S, Bornke F. Interactions of Xanthomonas type-III effector proteins with the plant ubiquitin and ubiquitin-like pathways. Front Plant Sci. 2014;5:736.

Valassakis C, Livanos P, Minopetrou M, Haralampidis K, Roussis A. Promoter analysis and functional implications of the selenium binding protein (SBP) gene family in Arabidopsis thaliana. J Plant Physiol. 2018;224-225:19-29.

Vriend G, Sander C. Detection of common three-dimensional substructures in proteins. Proteins. 1991;11:52-8.

Walter M, Chaban C, Schutze K, Batistic O, Weckermann K, Nake C, et al. Visualization of protein interactions in living plant cells using bimolecular fluorescence complementation. Plant J. 2004;40:428-38.

Wang M, Rui L, Yan H, Shi H, Zhao W, Lin JE, et al. The major leaf ferredoxin Fd2 regulates plant innate immunity in Arabidopsis. Mol Plant Pathol. 2018;19: 1377-90.

Wei CF, Kvitko BH, Shimizu R, Crabill E, Alfano JR, Lin NC, et al. A Pseudomonas syringae pv. tomato DC3000 mutant lacking the type III effector HopQ1-1 is able to cause disease in the model plant Nicotiana benthamiana. Plant J. 2007:51:32-46

Wright KM, Duncan GH, Pradel KS, Carr F, Wood S, Oparka KJ, et al. Analysis of the $N$ gene hypersensitive response induced by a fluorescently tagged tobacco mosaic virus. Plant Physiol. 2000;123:1375-86.

Wu L, Chen H, Curtis C, Fu ZQ. Go in for the kill: how plants deploy effectortriggered immunity to combat pathogens. Virulence. 2014;5:710-21.

Wu Y, Zhou JM. Receptor-like kinases in plant innate immunity. J Integr Plant Biol. 2013:55:1271-86.

Xu CC, Zhang D, Hann DR, Xie ZP, Staehelin C. Biochemical properties and in planta effects of NopM, a rhizobial E3 ubiquitin ligase. J Biol Chem. 2018;293: 15304-15.

Yamaguchi K, Yamada K, Ishikawa K, Yoshimura S, Hayashi N, Uchihashi K, et al. A receptor-like cytoplasmic kinase targeted by a plant pathogen effector is directly phosphorylated by the chitin receptor and mediates rice immunity. Cell Host Microbe. 2013;13:347-57.

Zhang H, Wang S. Rice versus Xanthomonas oryzae pv. oryzae: a unique pathosystem. Curr Opin Plant Biol. 2013;16:188-95.

Zou LF, Wang XP, Xiang Y, Zhang B, Li YR, Xiao YL, et al. Elucidation of the hrp clusters of Xanthomonas oryzae pv. oryzicola that control the hypersensitive response in nonhost tobacco and pathogenicity in susceptible host rice. Appl Environ Microbiol. 2006;72:6212-24.

Zurbriggen MD, Carrillo N, Tognetti VB, Melzer M, Peisker M, Hause B, et al. Chloroplast-generated reactive oxygen species play a major role in localized cell death during the non-host interaction between tobacco and Xanthomonas campestris pv. vesicatoria. Plant J. 2009:60:962-73.

\section{Ready to submit your research? Choose BMC and benefit from:}

- fast, convenient online submission

- thorough peer review by experienced researchers in your field

- rapid publication on acceptance

- support for research data, including large and complex data types

- gold Open Access which fosters wider collaboration and increased citations

- maximum visibility for your research: over $100 \mathrm{M}$ website views per year

At BMC, research is always in progress.

Learn more biomedcentral.com/submissions 